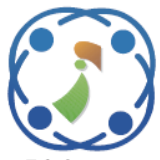

\title{
Velocity Trajectory Optimization with Time Management in Electric Vehicles
}

\author{
Waiard Saikong $^{1} \quad$ Chaiyut Sumpavakup $^{2} \quad$ Thanatchai Kulworawanichpong $^{1 *}$ \\ ${ }^{1}$ School of Electrical Engineering, Suranaree University of Technology, Nakhon Ratchasima 30000, Thailand \\ ${ }^{2}$ Research Centre for Combustion Technology and Alternative Energy - CTAE, \\ College of Industrial Technology, King Mongkut's University of Technology North Bangkok, Bangkok 10800, \\ Thailand \\ * Corresponding author’s Email: thanatchai@gmail.com
}

\begin{abstract}
An electric vehicle is one of the crucial technologies for transportation of passengers and goods to reduce the consumption of fossil fuel which is the main factor of air pollution. Currently, energy and power density of batteries are low when compared to fossil fuel, having an effect that electric vehicles have limited driving range. As a result, simulating velocity trajectory for optimal energy consumption for electric vehicle is necessary. This paper introduced the algorithm to determine optimal velocity trajectory or velocity profile to consume the least energy in the required cycle with the condition that the optimal velocity had to be similar to the profile of the reference velocity or the original velocity so that it was capable of driving in the traffic circumstance of the sample cycles. To find the optimal velocity with the least energy consumption, the simple vehicle model and mathematic approach to simulate optimal vehicle trajectory by particles swarm optimization (PSO) were used in this study. The route used in the study were divided into elementary driving cycles. The algorithm will simulate the acceleration and create the optimal velocity trajectory of each segment to find the trajectory that consumed the least energy regarding the condition of driving time determined by user. The result of the simulation found that the algorithm reduced the consumption of energy and maximum electric power with significance. That were $13.44 \%$ and $14.225 \%$ respectively when the electric vehicle was determined to arrive at the destination by 1 minute late.
\end{abstract}

Keywords: Electric vehicles simulation, Vehicle trajectory, Energy consumption, Optimization.

\section{Introduction}

Nowadays, fossil fuel is still the main fuel in transportation to freely transport goods and passengers for economic and social development. Generally, the world consumption of fuel is approximately 85 million barrels per day and $60 \%$ is used in transportation. All round the world, there are approximately 800 million private cars [1] and the number is increasing. In 2009, China is the biggest manufacturer and the biggest car market of the world leaving USA behind by manufacturing 13.79 million cars in the year and the trend of the supply and demand is rapidly rising every year. The aforementioned report indicates that transportation also has demand to consume more fuel which is limited resource. There will also be pollution emission from combustion leading to change of atmosphere and pollution in city [2] which are critical environmental problems. To solve the problem, it is suggested to use energy from other source or use energies from various sources for sustainable consumption. Additionally, there must be the least possible emission of pollution. The use of electric energy is the most flexible since it can be obtained from many power sources without emission to cause air pollution. However, the energy and power density of batteries are low when compared to fossil fuel [3] leading to the fact that electric car has limited driving range. Moreover, the rate of electrical power supply to traction motor is limited. Higher electrical power supply than the capacity of the batteries brings about exceeding heat and later leading to problem of safety [4]. As a result, batteries in present electric vehicle has to be unnecessarily big since it has to supply high 
electrical peak power. Therefore, simulation of optimal energy and power for electric vehicle is significant.

In general, vehicle trajectory model of each route is the unique driving characteristic which includes factors affecting movement profile such as traffic, types of vehicle, and the legal speed limit of each route. As a result, changing velocity trajectory model to simulate optimal rate must depend on traffic environment which was categorized into 2 types: open environment and closed environment [5]. Open environment is the traffic considerably affected by external factors such as heavy traffic on public road which has an effect on the velocity trajectory by other vehicles sharing the same road, traffic signs, and pedestrians. The adjustment to optimal velocity trajectory for application to authentic circumstance is difficult or impossible at all. Closed environment, on the other hand, is the studied traffic environment which is affected by only few external factors such as pathway in parks, pathway in zoos, streets in universities where driving is in closed area, and public road or highway with light traffic which can be considered a driving condition in closed environment. The adjustment of driving profile to save energy and apply to the studied can possibly be implemented in such environment.

There are several studies proposing electric vehicle driving by simulating energy saving velocity trajectory to increase driving range. For example, [6] presented an approach to plan an optimal velocity trajectory to simulate the velocity trajectory employing simple vehicle model and dynamic programming algorithm. The driving route was divided to equal segments of $10 \mathrm{~m}$. each. The algorithm simulated optimal velocity of each sections to find the point of minimum power usage regarding State of Charge: SOC and driving time. Additionally, [7] the simulation saved energy by reusing kinetic energy and potential energy by storing back to batteries when brake or drive downhill and then bringing the stored power to be used as driving energy. The result of the study revealed energy saving by $5 \%$ when compared to cruise controller with constant velocity set point. Furthermore, $[8,9]$ used probabilistic model to estimate the vehicle velocity trajectories by examining all possible sequences of modal activities (e.g., acceleration, deceleration, cruising, and idling) between consecutive data points from sparse position and vehicle velocity measurements. This model requires real-time traffic data from smartphones or cellular network to optimize powertrain operation strategy and vehicle speed trajectory [10].
In this paper, a new algorithm is proposed to simulate velocity trajectory of driving so that electric vehicles consumed optimum energy under the condition of the driving time determined by a driver. The simulated velocity trajectory was similar to original trajectory so that the newly simulated velocity was applicable to authentic use and applicable under the condition of the original traffic environment, both of open traffic environment or closed traffic environment. When the driver determined the time to reach the destination slower or spend more time to drive, the algorithm calculated optimal velocity trajectory by particles swarm optimization: PSO [11-14] for saving energy consumption and reached the destination by the appointed time. The simulation used the original velocity to be the upper bound velocity limit. This formulation was to compare consumption of energy and power when the driver determined to reach the destination by 30 seconds and 60 seconds late.

\section{Mathematical model and optimization formulation}

This part was to explain application of mathematic model to explain electrical vehicle movement to find power and energy consumption. After that examined acceleration with least energy consumption under the condition of the proposed vehicle trajectory. From Newton's second law of motion, the dynamic equation of vehicle movement is expressed by Eq. (1). The driving power was equal to the resistance power plus the dynamic power of acceleration of the vehicle as shown in Eq. (3) and Fig. 1 showing force acting on the vehicle.

$$
F_{t}-F_{r r}-F_{a d}-F_{r g}=M_{v} \frac{d V}{d t}
$$

From the equation of power [1],

$$
P_{e}=F_{t} \times V
$$

Eq. (1) and (2) can be written in the form of Eq. (3)

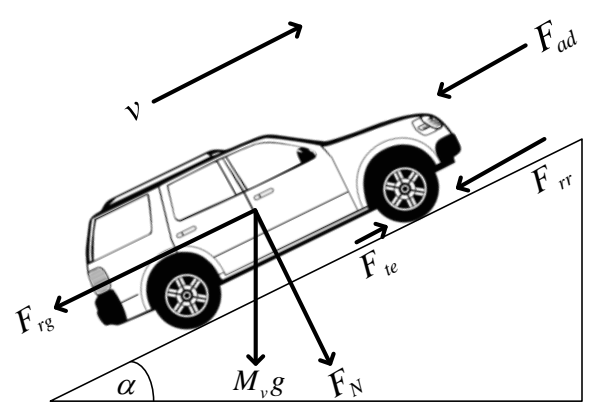

Figure.1 Free-body diagram of a vehicle on a gradient 


$$
P_{e}=\frac{V}{\eta_{t}}\left(F_{r r}+F_{a d}+F_{r g}+M_{v} \delta \frac{d V}{d t}\right)
$$

Suppose that a vehicle's velocity is $V$, a vehicle of mass $M_{v}$ is on a slope making an angle $\alpha$ to the horizontal as shown in Fig. 1, the vehicle motion is opposed by various forces, e.g. aerodynamic drag $\left(F_{a d}\right)$, gradient force $\left(F_{r g}\right)$, and rolling resistance force $\left(F_{r r}\right)[1,15] . \quad a$ is an acceleration and is called $\delta$ rotational inertia factor [3]. The power loss in transmission, motor drive loses including power losses in the electrics are represented by the efficiency as shown in the table 1. The Eq. (3) can be written as Eq. (4).

$$
P_{e}=\frac{V}{1000 \eta_{t}}\left(\begin{array}{c}
M_{v} g f_{r} \cos \alpha+\frac{1}{2} \rho_{a} C_{D} A_{f} V^{2} \ldots \\
+M g f_{r} \sin \alpha+M_{v} \delta \frac{d V}{d t}
\end{array}\right)
$$

Electric vehicle energy consumption in kilowatt unit $(\mathrm{kW})$ can be calculated by integration of the power consumption and can be expressed as Eq. (5), [3]

$$
E_{e}=\int_{t_{0}}^{t_{\text {final }}} P_{e} d t
$$

where $E_{e}$ is the net energy consumption from batteries.

In order to optimise energy consumption using the particles swarm optimization (PSO). The objective function and constrain are shown in Eq. (6),

$$
\begin{aligned}
\operatorname{Min} f\left(E_{e}\right) & =\operatorname{Min}\left(E_{0}-E_{e}\left(a_{1}, a_{2}\right)\right) \\
\text { Subject to: } & 0 \leq a_{1} \leq a_{\text {ref_max } 1} \\
& a_{\text {ref_max_br }} \leq a_{2} \leq a_{\text {ref_max } 2} \\
& \left(t_{1}+t_{2}+\cdots+t_{n}\right) \leq t_{\text {final }} \\
& t_{\text {final }}=t_{\text {ref }}+t_{\text {late }}
\end{aligned}
$$

where $E_{0}$ is the initial energy in an energy storage system, $a_{1}$ and $a_{2}$ are acceleration parameters that are used in the algorithm in methodology section. $a_{\text {ref_max_br }}$ is a deceleration parameter that is used in braking period, $a_{\text {ref_maxl }}$ is a maximum acceleration parameter of the reference route that is used in acceleration period and $a_{\text {ref_max } 2}$ is a maximum acceleration (or deceleration) parameter of the reference route that is used in constant velocity and coasting period. $\left(t_{1}+t_{2}+\ldots+t_{n}\right)$ are the driving time in elementary cycles, $t_{\text {final }}$ is the overall driving time, $t_{\text {ref }}$ is the driving time of the reference route and $t_{\text {late }}$ is the late time constraint as driver input. An optimization process has acceptable tolerance of less than $10^{-6}$.
Table 1 Parameters used for simulation

\begin{tabular}{|l|c|c|c|}
\hline \multicolumn{1}{|c|}{ Parameters } & Symbol & Unit & Value \\
\hline Vehicle Mass & $M_{v}$ & $\mathrm{~kg}$ & 1500 \\
\hline $\begin{array}{l}\text { Rolling resist. } \\
\text { coefficient }\end{array}$ & $f_{r}$ & - & 0.010 \\
\hline $\begin{array}{l}\text { Aerodynamic drag } \\
\text { coefficient }\end{array}$ & $C_{D}$ & - & 0.300 \\
\hline Front area & $A$ & $\mathrm{~m}^{2}$ & 2.200 \\
\hline Air density & $r_{0}$ & $\mathrm{~kg} / \mathrm{m}^{3}$ & 1.1455 \\
\hline $\begin{array}{l}\text { Gravitational } \\
\text { acceleration }\end{array}$ & $g$ & $\mathrm{~m} / \mathrm{s}^{2}$ & 9.810 \\
\hline Vehicle's velocity & $V$ & $\mathrm{~m} / \mathrm{s}$ & - \\
\hline $\begin{array}{l}\text { Rotational inertia } \\
\text { factor }\end{array}$ & $\delta$ & - & 1.084 \\
\hline Efficiency & $\eta_{t}$ & - & 0.960 \\
\hline $\begin{array}{l}\text { Net energy } \\
\text { consumption }\end{array}$ & $E_{e}$ & $\mathrm{kWh}$ & - \\
\hline $\begin{array}{l}\text { Initial energy in an } \\
\text { energy storage }\end{array}$ & $E_{0}$ & $\mathrm{kWh}$ & - \\
\hline $\begin{array}{l}\text { Vehicle } \\
\text { acceleration }\end{array}$ & $\frac{d V}{d t}$ or $a$ & $\mathrm{~m} / \mathrm{s}^{2}$ & - \\
\hline
\end{tabular}

\section{Simulation verification}

Mathematical model must be undergone investigation and verification to verify whether the simulation and the result of the simulation was reliable. This section was to compare the result of the simulation from the previously mentioned mathematical model. It was calculation using an iterative process and electric vehicle simulation for energy management and design: EVSED which is developed to compare the results with reliable academic document [3]. The comparison of traction energy, basic data of electric vehicle used in the simulation as shown in Table 1, and data of routes and driving cycles. The comparison was made with 4 routes which were LA92, US06 and New York City Cycle (NYCC) which were the standard route in the simulation [16]. The routes parameters were shown in Table 2 and velocity trajectory and time of driving cycle were presented in Fig. 2 to 5 respectively and the results of the simulation were compared in Table 3. The last route was the field data measurement by collecting energy consumption rate from CAN (Control Area Network) and GPS (Global Positioning System) in a provincial electricity authority electric zero emission bus (PEA-Zebus), developed by Suranaree University of Technology (SUT). The data sets were recorded every 1 second via CAN (Control Area Network) bus as shown in Fig. 6. The bus was driven for $12.54 \mathrm{~km}$ around SUT route. Then the result was compared to the result from the simulation using EVSED program using input data from GPS and the results of the simulation were presented in Fig. 6 and Table 4. 
Table 2. Route parameter in simulation comparison

\begin{tabular}{|l|c|c|c|}
\hline \multicolumn{1}{|c|}{ Route parameters } & LA92 & US06 & NYCC \\
\hline Max. speed $(\mathrm{km} / \mathrm{h})$ & 108.15 & 129.23 & 44.58 \\
\hline Ave. speed $(\mathrm{km} / \mathrm{h})$ & 39.60 & 77.84 & 11.43 \\
\hline $\begin{array}{l}\text { Traveling distance per } \\
\text { cycle }(\mathrm{km})\end{array}$ & 15.80 & 12.89 & 1.89 \\
\hline Driving time $(\mathrm{s})$ & 1435 & 596 & 598 \\
\hline Max. acceleration $\left(\mathrm{m} / \mathrm{s}^{2}\right)$ & 3.08 & 3.75 & 2.68 \\
\hline Max. deceleration $\left(\mathrm{m} / \mathrm{s}^{2}\right)$ & -3.93 & -3.08 & -2.64 \\
\hline
\end{tabular}

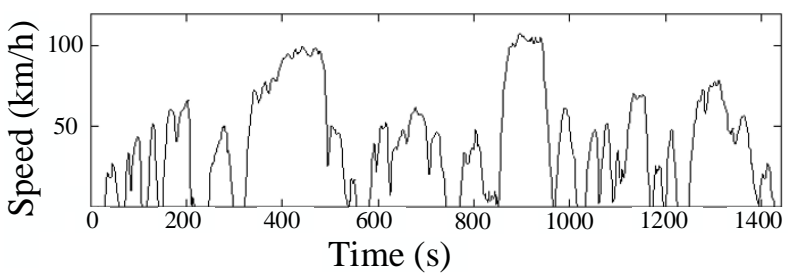

Figure. 2 Velocity trajectory and time of LA92 route

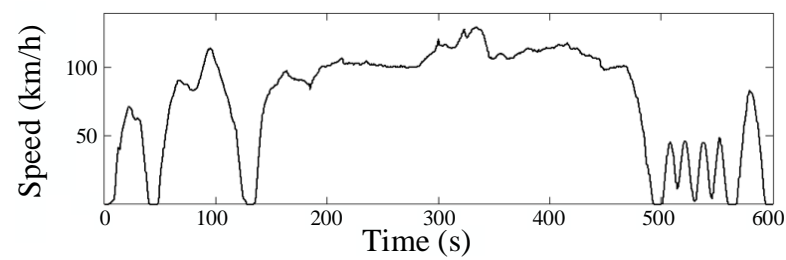

Figure. 3 Velocity trajectory and time of US06 route

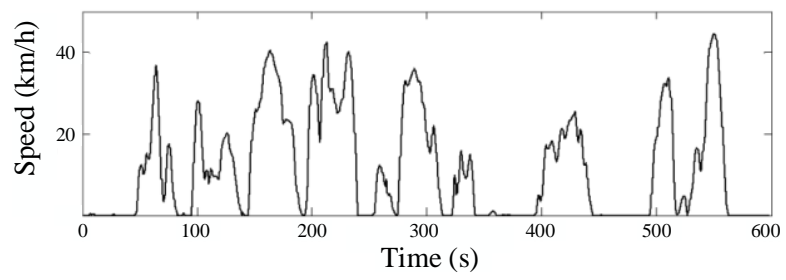

Figure. 4 Velocity trajectory and time of NYCC route

LA92 route, when compared to driving in urban area such as NYCC route, had higher maximum speed and average speed, but had less stops per distance and also had higher maximum acceleration.

US06 route was the route with the highest acceleration of all the routes in the test drive route used to compare in this study. This driving in the simulation represented aggressive driving.

NYCC route used low speed and often had stopand-go traffic which was the unique characteristic of driving in city.

Table 3. Comparison of traction energy

\begin{tabular}{|l|c|c|c|}
\hline \multirow{2}{*}{$\begin{array}{c}\text { Driving } \\
\text { cycles }\end{array}$} & \multicolumn{2}{|c|}{ Traction energy (kWh) } & \multirow{2}{*}{ \% Error } \\
\cline { 2 - 3 } LA92 & Ref. $^{*}$ & EVSED & \\
\hline US06 & 2.3559 & 2.3577 & 0.0764 \\
\hline NYCC & 0.2655 & 2.2490 & 0.7283 \\
\hline
\end{tabular}

Remark: Ref. refers to the test result from [3]
Table 4. Measurement of energy consumption and calculation result

\begin{tabular}{cccc}
\hline \multirow{2}{*}{$\begin{array}{c}\text { Driving } \\
\text { cycle }\end{array}$} & \multicolumn{2}{c}{ Traction energy (kWh) } & \multirow{2}{*}{$\%$ Error } \\
\cline { 2 - 3 } & CAN data & EVSED & \\
\hline SUT Route & 13.347 & 13.458 & 0.832 \\
\hline
\end{tabular}

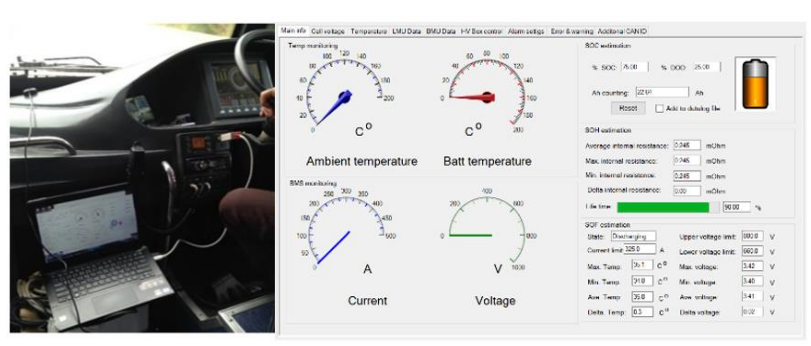

Figure. 5 Data measuring and logging via CAN bus

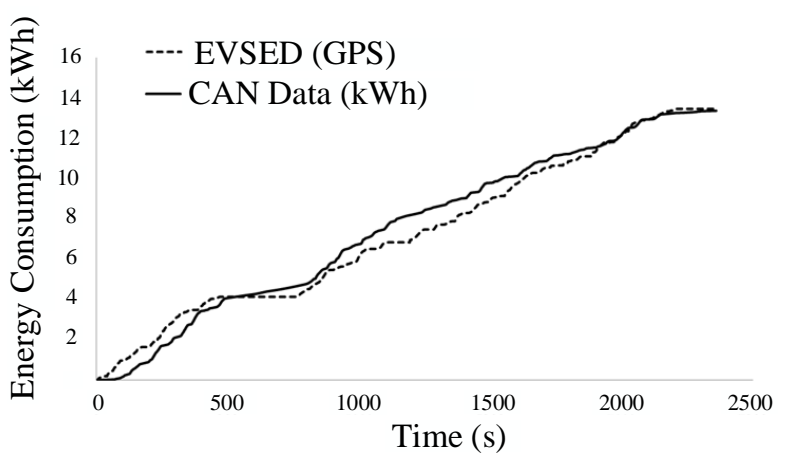

Figure. 6 Data measurement of energy consumption and simulation result of SUT route

The result of the simulation in Table 3 revealed that the maximum deviation was no more than $0.728 \%$ and the minimum deviation was $0.0764 \%$. The result of verification by the real filed measurement data in Table 4 presented deviation at only $0.832 \%$. This can be assumed that the calculation algorithm, test of electric vehicle for energy management, and EVSED design were accurate and reliable. Therefore, EVSED program that was developed by SUT research team was appropriate to employ in this study for energy management system and investigating electrical energy consumption of the electric vehicle which was reported in this article.

\section{Testing route and simulation}

This article used UNECE Reg.101 [17] as the reference route in the simulation which consisted of 2 cycles: urban driving cycle and extra urban driving cycle. The researcher also divided the cycles into 13 elementary driving cycles. Each elementary cycle referred to the position from parking or the position where velocity was zero to the next position where the velocity was zero again. As shown in Fig. 7, the data of the simulating routes are as followed: total 


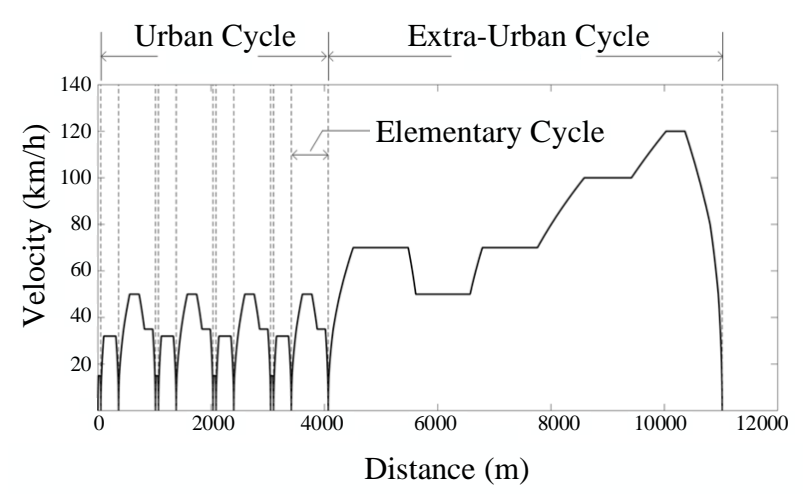

Figure. 7 The UNECE Reg.101 testing route velocity profile

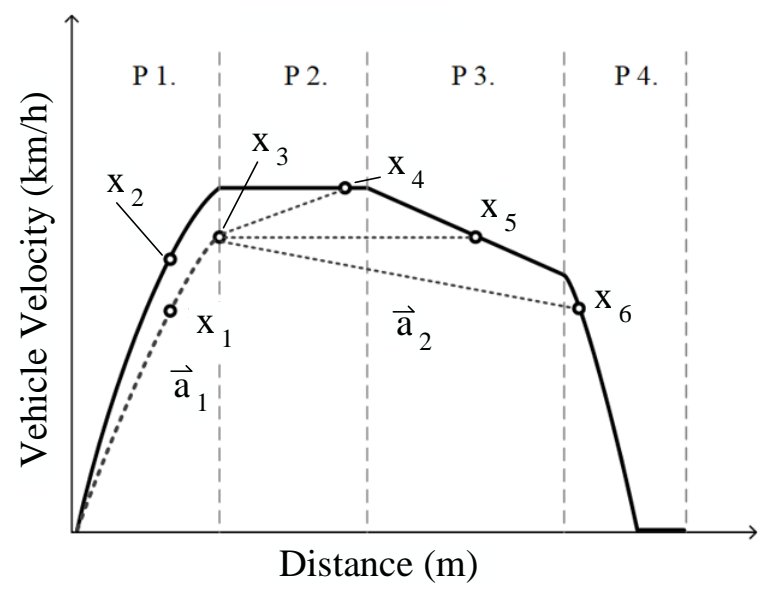

Figure. 8 Vehicle velocity profile cycle

distance $11,022 \mathrm{~m}$. , maximum velocity $120 \mathrm{~km} / \mathrm{h}$, average velocity $33.6 \mathrm{~km} / \mathrm{h}$, and driving time 1,180 seconds (s).

In this paper, one elementary cycle velocity profile of electric vehicle can be categorized into 4 segments as shown in Fig. 8. Part $1-\mathrm{P} 1$. (segment 1.) was the segment for accelerate velocity when it was accelerated to one point and entered part 2. Part $2-\mathrm{P} 2$ (segment 2.) was the stage of constant velocity or zero acceleration. Part $3-\mathrm{P} 3$ was the segment when the electric vehicle was moving by coasting or also using brake to slow down the acceleration. The last segment was Part 4 (P 4) where braking was used to lower velocity to zero or to stop. Authentic driving may consist of Part 2 and Part 3 or either one. 1 route cycle consisted of many elementary driving cycles. Shown in Fig. 7 was the test-driving route cycle UNECE Reg. 101 which consisted of 13 elementary cycles.

An algorithm is created to discover new velocity trajectory so that electric vehicle consumed optimum energy under the condition of time determined by drivers. The velocity profile had to resemble to original trajectory so that the newly created trajectory was applicable to the driving condition of the original route, for both open and closed traffic environment. When drivers determined to arrive at the destination slower or spend more time in driving, the algorithm would calculate the optimal velocity for saving energy consumption and arrived at the destination by the time determined. The algorithm used the original velocity profile as the upper bound velocity limit. In other words, the newly created velocity profile was not faster than the original velocity profile. In Fig. 8, the velocity profile in solid line was the original velocity trajectory while the dashed line was the new velocity trajectory. In this study, the test was a comparison of power and energy consumption when the driver determined to arrive at the destination by 30 seconds and 60 seconds late. The process of creating new velocity trajectory composes of 4 steps as follows.

\section{Steps in Creating the New Velocity Profile Algorithm}

Step 1. Categorized velocity profile of original route into elementary cycles as shown in Fig. 7 and presented the process in Fig. 9

Step 2. Investigated each cycle in Part 1 and find optimal acceleration $\left(\vec{a}_{1 x}\right)$ by finding the result in PSO. The condition was that $\vec{a}_{1 x}$ was the acceleration of segment 1 of the elementary cycle number $\mathrm{x}$ as shown in Fig. 9 with the condition that the result of vehicle velocity calculation obtained from the simulation must not exceed the original velocity profile as in Fig. 8 at $\mathrm{x}_{1}$ where the minimum velocity was lower than $\mathrm{x}_{2}$ on the line of original velocity which was used as the limit of the maximum velocity. Time spent in driving was not exceed the time determined by the driver. (This test was to compare power and energy consumption when the driver determined to arrive at the destination by 30 seconds and 60 seconds late.)

Step 3. Investigated segment 2 and segment 3 together to find acceleration $\left(\vec{a}_{2 x}\right)$ when $\vec{a}_{2 x}$ was the acceleration of segment 2 of elementary cycle number $x$ as shown in Fig. 9. Consider $x_{3}$ in Fig. 8 by the same approach as in step 2 but with wider limit. The acceleration obtained was possible to be positive (increasing velocity), zero (steady velocity), or negative (decreasing velocity). This contributed to the 3 possible velocity profiles as followed from $\mathrm{x}_{3}$ to $\mathrm{x}_{4}$, from $\mathrm{x}_{3}$ to $\mathrm{x}_{5}$ and from $\mathrm{x}_{3}$ to $\mathrm{x}_{6}$ as shown in Fig. 8 .

Step 4. Use $\vec{a}_{1 x}$ and $\vec{a}_{2 x}$ to create the velocity profile under the constrains. when the new velocity profile was equal to the original velocity profile at the same position (at equal distance) and was the position of the acceleration of the original velocity profile 


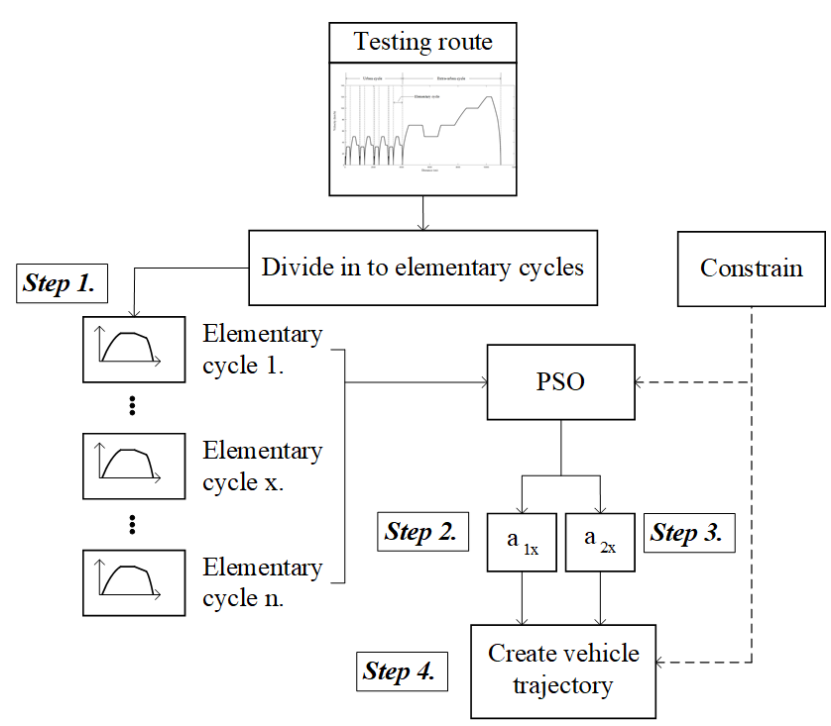

Figure. 9 Steps in creating new velocity trajectory

(used as reference velocity profile) was lower than $\vec{a}_{1 x}$ and $\vec{a}_{2 x}$, then fixed the new velocity profile to be equal to the original velocity profile. When entering segment 4 where braking was needed, the brake profiles were the same and always stop at the same position. The mentioned condition was as in Eq. (4). Then repeated step 2 to step 4 for all elementary driving cycles all along the route.

\section{Testing results}

The test results consist of 2 topics, the comparison of the proposed algorithm to cruise control algorithm and the optimal velocity trajectory of route UNECE Reg. 101 to compare the energy and peak power consumption. movement simulation to compare the proposed algorithm of the researcher to cruise control algorithm which is available in general automobiles. The reference velocity profile (bold black line as in figure 10) has maximum speed at $90 \mathrm{~km} / \mathrm{h}$, distance of $4690.7 \mathrm{~m}$, and driving time of 214 seconds. The simulation creates the velocity profile that arrives late at no more than 30 seconds. The results of energy and maximum power consumption when driving are then compared. The result of the simulation can be seen in table 5. From table 5, it is found that energy consumptions of the proposed algorithm and cruise control algorithm decrease when compared to reference velocity profile by $18.345 \%$ and $12.589 \%$, respectively. The maximum power of the proposed algorithm decreases by $21.872 \%$ and $12.784 \%$ for the cruise control algorithm. When compared to the reference velocity, the algorithm that the researcher proposed consumes less driving energy and is able to decrease more maximum electrical power. It can be concluded that the proposed algorithm is more advanced in energy and power consumption than cruise control algorithm in general automobile.

The testing results of the velocity trajectory of route UNECE Reg. 101 as the reference route which was the route consisting of the characteristics of urban driving cycle and extra-urban driving cycle as shown in Fig.7 and used as the upper bound velocity limit were divided in to 2 cases. Case 1 determined the car to arrive 30 second late and case 2 determined the car to arrive 60 seconds late from the time of the reference route. Then compared the result of the simulations and reference velocity trajectory as shown in Fig. 11 and 12.

In Fig. 11 in the stage of acceleration (segment 1.), it was found that the adjusted optimal velocity trajectory in the case of arriving at the destination 30 and 60 seconds late presented changes of subsequent decreasing velocity and acceleration when compared to the velocity trajectory of the reference route. The acceleration can be observed in Table 6. In part 2 (segment 2.), when investigating the urban route, the elementary cycle 1 and elementary cycle 2 presented increasing deceleration when arriving at the destination spending longer time. In the elementary cycle 3 and extra urban driving or the last elementary cycle presented similar trends that was deceleration $\left(\vec{a}_{2}\right)$ of velocity trajectory with 30 s late profile presented the highest value, in other words, the most deceleration. Additionally, the velocity trajectory case 1 (30s late) presented the same value as the reference velocity trajectory. In the velocity trajectory case 2 , other parts presented the lower velocity than the reference velocity trajectory but they presented imitation of velocity trajectory. Fig. 12 showed changes of velocity trajectory and time of each case.

Table 5. Results of comparison of algorithms for optimal velocity profile

\begin{tabular}{|l|c|c|c|c|}
\hline \multicolumn{1}{|c|}{ Speed profile } & $\begin{array}{c}\text { Energy consumption } \\
(\mathrm{kWh})\end{array}$ & $\begin{array}{c}\text { Peak power } \\
(\mathrm{kW})\end{array}$ & $\begin{array}{c}\text { Average speed } \\
(\mathrm{km} / \mathrm{h})\end{array}$ & $\begin{array}{c}\text { Max. speed } \\
(\mathrm{km} / \mathrm{h})\end{array}$ \\
\hline Reference velocity profile & 0.837 & 87.522 & 78.542 & 90.000 \\
Proposed algorithm & 0.681 & 68.379 & 68.196 & 82.563 \\
Cruise control algorithm & 0.729 & 76.333 & 70.830 & 80.006 \\
\hline
\end{tabular}




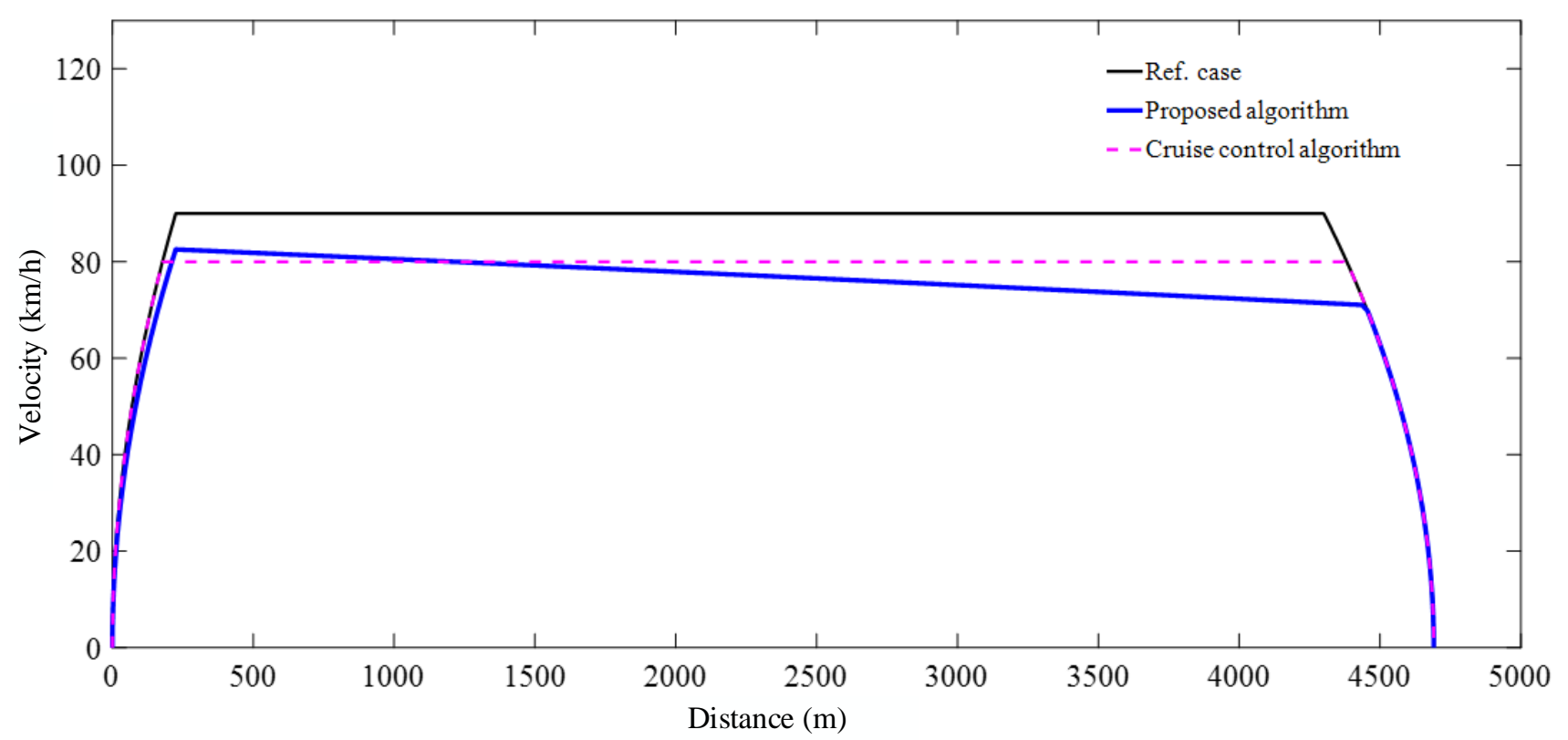

Figure. 10 Comparison of velocity profile from the proposed algorithm and cruise control algorithm

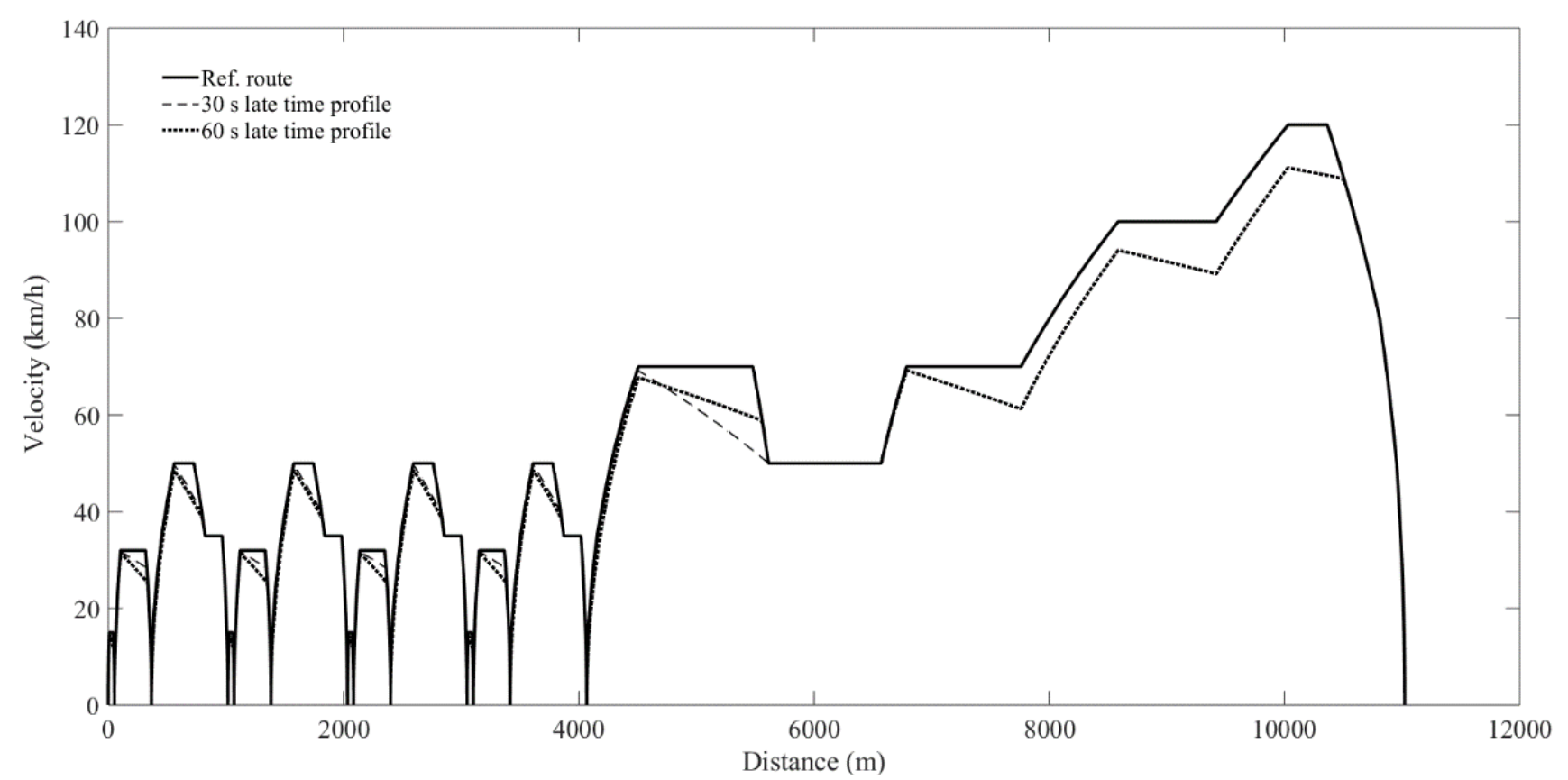

Figure. 11 Results of simulations and comparison of velocity trajectory and position 


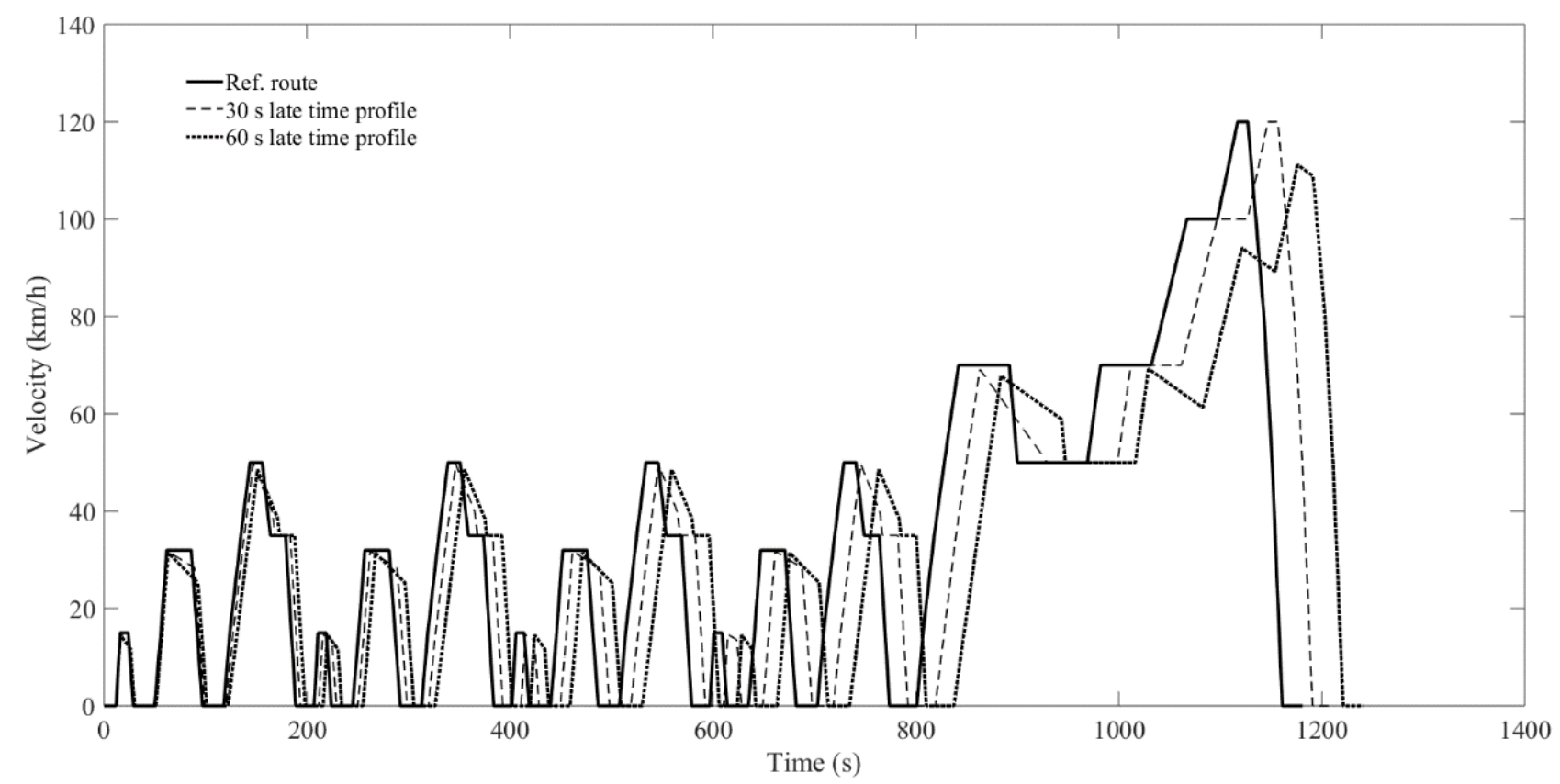

Figure. 12 Results of simulations and comparison of velocity trajectory and time

Table 6 Accelerating parameters comparison

\begin{tabular}{|c|c|c|c|c|c|}
\hline \multirow{4}{*}{ Route information } & ref. route & 30 s late & 60 s late \\
\hline \multirow{4}{*}{ Urban route } & \multirow{3}{*}{ The first elementary cycle } & $\mathrm{a}_{1}$ & 1.042 & 0.991 & 0.986 \\
\cline { 3 - 6 } & \multirow{2}{*}{ The second elementary cycle } & $\mathrm{a}_{2}$ & 0.000 & -0.033 & -0.080 \\
\cline { 3 - 6 } & $\mathrm{a}_{1}$ & 0.787 & 0.770 & 0.752 \\
\cline { 3 - 6 } & \multirow{2}{*}{ The third elementary cycle } & $\mathrm{a}_{2}$ & 0.000 & -0.035 & -0.058 \\
\cline { 3 - 6 } & & $\mathrm{a}_{1}$ & 0.694 & 0.593 & 0.470 \\
\hline \multirow{2}{*}{ Extra-urban route } & \multirow{2}{*}{ The forth elementary cycle } & $\mathrm{a}_{1}$ & 0.000 & -0.145 & -0.140 \\
\cline { 3 - 6 } & & $\mathrm{a}_{2}$ & 0.694 & 0.480 & 0.408 \\
\hline
\end{tabular}

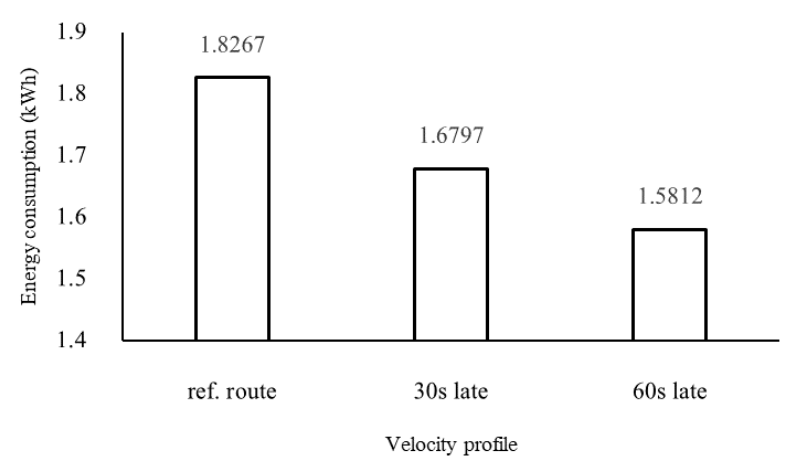

Figure. 13 Energy consumed in driving of velocity profile

In Fig. 13, it was found that consumption of power in driving of electric vehicle in the new velocity profile presented lower value than velocity profile of the reference route in both case 1 and case 2 . In case 1 which fixed that the electric vehicle arrived at the destination 30 second late consumed energy of $46.0394 \mathrm{kWh}$ or decreasing by $8.0473 \%$ of energy used in reference route. In case 2 which the researcher fixed that the electric vehicle arrived at the destination 60 second late consumed energy of
$39.968 \mathrm{kWh}$ or decreasing by $13.44 \%$. Not only that the energy consumption was decreased, but the maximum power was decreased with significance. Fig. 14 showed the comparison of maximum electric power of each velocity trajectory. Case 1 consumed maximum electrical power in driving at $46.0394 \mathrm{~kW}$ or decreasing by $1.195 \%$ and case 2 consumed peak electrical power at $39.968 \mathrm{~kW}$ or decreasing by $14.225 \%$

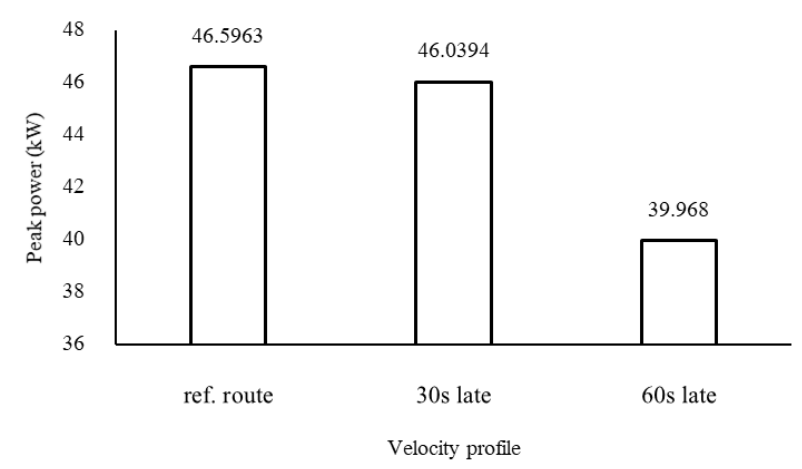

Figure. 14 Peak power consumed in driving of velocity profile 


\section{Conclusion}

The strength of this study is the improvement of optimal velocity profile under the condition of late arrival at the destination. The optimal velocity profile for the late arrival is crucial. The proposed procedure in the article presented the improvement from optimal velocity profile that brings about the results that the automobile arrives at the appointed time and consumes less energy and maximum driving power than cruise control algorithm, a control algorithm that automobiles generally have nowadays. The proposed algorithm was flexible and drivers can input the required late time to arrive at the destination. Moreover, the algorithm can reduce energy consumption with significance. It was an alternative efficient choice to use the algorithm to reduce energy consumption. The results revealed that the algorithm can reduce electrical peak power and was attributable to prolonging of batteries expiration. In terms of energy storage system, the algorithm supported reduction batteries size since the limitation of lower electrical power density had an effect on the design to be oversized and overweighed to support highest electrical power supply. Additionally, the velocity trajectory resulted from the proposed algorithm resembled the original velocity trajectory or the reference velocity trajectory and allowed drivers to be able to drive in the optimal velocity trajectory so that it is applicable to traffic conditions or environments similar to the reference velocity profile.

\section{References}

[1] M. Chris, M.A. Masrur, and D.W. Gao, "Hybrid Vehicles Principles and Applications with Practical Perspectives", John Wiley \& Sons Inc. Publication, Singapore, 2011.

[2] U.S. Environmental Protection Agency, Inventory of U.S. Greenhouse Gas Emissions and Sinks: 1990 - 2014. Final Report. EPA 430R-16-002, 2016.

[3] M. Ehsani, Y. Gao, and A. Emadi, "Modern electric, hybrid electric, and fuel cell vehicles: fundamentals, theory, and design", Power Electronics and Applications Series (2nd), CRC press, 2010.

[4] A. Davide, "Battery management systems for large lithium-ion battery packs", Artech house Norwood, MA, 2010.

[5] Y.J. Jang, E.S. Suh, and J.W. Kim, "System Architecture and Mathematical Models of Electric Transit Bus System Utilizing Wireless Power Transfer Technology", IEEE Systems Journal, Vol. 10, No. 2, pp. 495-506, 2016.
[6] S. Rohkämper, M. Hellwig, and W. Ritschel, "Energy optimization for electric vehicles using dynamic programming", In: Proc. of International Conference on Research and Education in Mechatronics, pp. 1-5, 2017.

[7] Tv. Keulen, Bd. Jager, D. Foster, and M. Steinbuch, "Velocity trajectory optimization in Hybrid Electric trucks", In: Proc. of American Control Conference, pp. 5074-5079, 2010.

[8] P. Hao, K. Boriboonsomsin, G. Wu, and MJ. Barth, "Modal Activity-Based Stochastic Model for Estimating Vehicle Trajectories from Sparse Mobile Sensor Data", IEEE Transactions on Intelligent Transportation Systems, Vol.18, No. 3, pp. 701-711, 2017.

[9] X. Shan, P. Hao, X. Chen, K. Boriboonsomsin, G. Wu, and M.J. Barth, "Probabilistic model for vehicle trajectories reconstruction using sparse mobile sensor data on freeways", In: Proc. of IEEE 19th International Conference on Intelligent Transportation Systems, pp. 689-694, 2016.

[10] X. Qi, G. Wu, P. Hao, K. Boriboonsomsin, and MJ. Barth, "Integrated-Connected Eco-Driving System for PHEVs With Co-Optimization of Vehicle Dynamics and Powertrain Operations", IEEE Transactions on Intelligent Vehicles, Vol. 2, No. 1, pp. 2-13, 2017.

[11] I.C. Trelea, "The particle swarm optimization algorithm: convergence analysis and parameter selection", Information Processing Letters, Vol. 85, No. 6, pp. 317-325, 2003.

[12] J. Kennedy and R. Eberhart, "Particle swarm optimization", In: Proc. of the 4th IEEE International Conference on Neural Networks, pp. 1942-1948, 1995.

[13] R. Eberhart and J. Kennedy, "A new optimizer using particle swarm theory", In: Proc. of the 6th International Symposium on Micro Machine and Human Science, pp. 39-43, 1995.

[14] Y.L. Zheng, L.H. Ma, L.Y. Zhang, and J.X. Qian, "On the convergence analysis and parameter selection in particle swarm optimization", In: Proc. of International Conference on Machine Learning and Cybernetics, pp. 1802-1807, 2003.

[15] W. Saikong, and T. Kulworawanichpong, "Comparative Study of Energy Consumption for Electric Vehicles with Various On-board Energy Storage Systems", Energy Procedia, Vol. 138, pp. 81-86, 2017.

[16] United States Environmental Protection Agency, “Dynamometer Drive Schedules", [online], 2018, Available: https://www.epa.gov/vehicle-and- 
fuel-emissions-testing/dynamometer-driveschedules\#vehicleDDS

[17] The United Nations Economic Commission for Europe, "UN Vehicle Regulations - 1958. UN Regulation No. 101 - Rev.3", [online], 2018, Available: https://www.unece.org/?id=39146 